\title{
Statistical physics of dyons and quark confinement
}

\author{
Dmitri Diakonov and Victor Petrov
}

\author{
Petersburg Nuclear Physics Institute, Gatchina 188300, St. Petersburg, Russia
}

\begin{abstract}
We present a semiclassical approach to the $S U(N)$ Yang-Mills theory whose partition function at nonzero temperatures is approximated by a saddle point - an ensemble of an infinite number of interacting dyons of $N$ kinds. The ensemble is governed by an exactly solvable $3 d$ quantum field theory, allowing calculation of correlations functions relevant to confinement. We show that known criteria of confinement are satisfied in this semiclassical approximation: (i) the average Polyakov line is zero below some critical temperature, and nonzero above it, (ii) a quark-antiquark pair has linear rising potential energy, (iii) the average spatial Wilson loop falls off exponentially with the area, (iv) $N^{2}$ gluons are canceled out from the spectrum, (v) the critical deconfinement temperature is in good agreement with lattice data.

Using the same approximation, we find confinement for the exceptional gauge group $G(2)$ and a first-order deconfinement transition, also in agreement with lattice findings.
\end{abstract}

Keywords: Quantum Chromodynamics, monopole, holonomy, semiclassical approximation, confinement-deconfinement phase transition PACS: 11.15.-q 11.10.Wx 11.15.Kc 12.38.Aw

\section{INTRODUCTION}

Quark confinement is one of the most puzzling phenomena in modern physics. It is widely believed (and supported by numerical simulations on a lattice) that if one attempts to separate a probe quark from an antiquark, or a quark from two other quarks in a proton, a force of about 14 tons (called the string tension) pulls it back, and this force does not decrease with the separation! Although long-range forces can be found in condensed matter physics (e.g. in the Ising model), it is for the first time in history we encounter such a phenomenon in a local microscopic theory, and it cries for an explanation. However, after 35 years [1] of Quantum Chromodynamics (QCD) not only an $a b$ initio mathematical derivation of the " 14 tons" is absent but there is still no consensus on what is the qualitative mechanism of quark confinement.

Today we know for sure that nuclear and subnuclear physics is governed by QCD - a local renormalizable non-Abelian gauge theory of "colored" quarks interacting with gluons being analogs of photons. Therefore, the problem of confinement is, at least, formulated mathematically: it must be a property of the quantum YangMills (YM) theory. Non-Abelian gauge invariance requires that gluons interact, even in the absence of quarks. In this paper we consider the pure YM theory with no dynamical quarks, also called the "pure glue" theory. However, quark sources or probes can be inserted to test certain YM correlation functions of interest, in particular we shall be interested in the correlation function of Polyakov lines and the average of large Wilson loops: these are quantities that measure the force between probe quarks.

Gluons are massless due to gauge invariance, and the only freedom a theorist has in a pure glue theory is the choice of the gauge group. It is $S U$ (3) for the real world, but it is helpful to consider Yang-Mills theories based on other Lie groups, in particular $S U(N)$ at any $N$. (At the end of the paper we consider also the exceptional $G(2)$ group for reasons explained there.) The scaling of most physical observables with $N$ at large $N$ can be found from simple $N$-counting rules, therefore considering arbitrary $S U(N)$ gauge groups provides a powerful check.

The gauge coupling constant $\alpha_{s}$, the analog of the QED coupling $\alpha \simeq 1 / 137$, is in fact not a constant and hence not a parameter that one can choose at will: it "runs" as function of the characteristic momentum at hand. Owing to asymptotic freedom, it is small at large and large at small momenta. As in any other asymptotically free theory with no explicit scale parameter, the "dimensional transmutation" occurs in the YM theory: an exponentially large correlation length $\xi$ appears, being the renormalization-invariant combination of the ultraviolet cutoff (e.g. the lattice spacing $a$ ) and the bare coupling constant $\alpha_{s}(a)$ given at that cutoff,

$$
\xi \equiv \frac{1}{\Lambda}=a \exp \left(\frac{3}{11} \frac{1}{\lambda(a)}\right), \quad \lambda(a)=\frac{\alpha_{s}(a) N}{2 \pi}
$$

where $\lambda(a)$ is the so-called 't Hooft bare coupling constant; it does not depend on $N$ at large $N$. $\Lambda$ has the dimension of mass; it is called the YM scale parameter and it actually defines all dimensional quantities in the theory. The deconfinement phase transition temperature $T_{c}$ is proportional to $\Lambda$ and the string tension is proportional to $\Lambda^{2}$ by dimensions. All dimensionless quantities are, generally speaking, of the order of unity, hence it is a strong-coupling problem from the start. It makes the theorist's life hard. 
Unfortunately, QCD will hardly be ever proved to be an exactly solvable quantum field theory, even in the large $N$ limit. Therefore, one can either do exact calculations in a theory that has more symmetries but is not our world (e.g. considering supersymmetric versions of QCD), or work with QCD but make approximations. The first is useful as a theoretical laboratory, the second is necessary to understand semi-quantitatively the key phenomena, to explain experimental data, and to make predictions.

An approximation is considered to be legitimate if there is a systematic way of improving its accuracy. The semiclassical approach which we develop below, belongs to this category. One chooses a saddle-point classical field and then has to take into account quantum fluctuations about it. Part of the fluctuations are ultra-violet and are thus the same as in empty space. Their role is to renormalize the bare coupling constant; at this point the YM scale parameter (1) emerges. What is left, is a series in 't Hooft's running coupling $\lambda$ coming from loop expansion in the background of classical configurations. The running coupling is evaluated at the maximal momentum in the problem, be it temperature or the average density of classical configurations. Speaking generally, such expansion parameter is of the order of unity, however numerically it turns out to be small: $\lambda$ is between $\frac{1}{4}$ at zero temperature and $\frac{1}{7}$ near $T_{c}$. Therefore, in the whole range of temperatures within the confining phase the semiclassical approximation is expected to yield an accuracy of $15-25 \%$, already in the 1-loop approximation (provided the saddle point is chosen correctly!) with a potential for rapid improvement when higher loops are taken into account. We shall see, however, that the actual accuracy can be much better than this estimate. It is not a too big price to pay if confinement, the most challenging riddle in 35 years, is explained in simple terms.

We consider the pure Yang-Mills theory based on the $S U(N)$ gauge group in a broad range of temperatures between 0 and $T_{c}$, the deconfinement phase transition temperature. Although the formalism we use is designed for nonzero $T$, we shall see that the physical observables we find (such as the string tension) have a finite limit when $T \rightarrow 0$.

Confinement, as we understand it today and learn from lattice experiments with a pure glue theory, has in fact many facets, and all have to be explained. For example, in a general $S U(N)$ group one can consider "quarks" in various irreducible representations. From the confinement viewpoint all representations are characterized by the phase it acquires under the gauge transformation from the group center. The representation is said to have " $N$-ality $=k$ " if the phase is $\frac{2 \pi k}{N}$. Let us formulate mathematically the main confinement requirements that need to be satisfied:

- the average Polyakov line in any $N$-ality nonzero representation of the $S U(N)$ group is zero below $T_{c}$ and nonzero above it

- the potential energy of two static color sources (defined through the correlation function of two Polyakov lines) asymptotically rises linearly with the separation; the slope called the string tension depends only on the $\mathrm{N}$-ality of the sources

- the average of the spatial Wilson loop decays exponentially with the area spanning the contour; at vanishing temperatures the spatial ("magnetic") string tension has to coincide with the "electric" one, for all representations

- the mass gap: no massless gluons should be left in the spectrum.

Remarkably, all these requirements are satisfied already in a semiclassical approximation if one uses an ensemble of dyons as a saddle point in the Yang-Mills partition function [2].

\section{YANG-MILLS THEORY AT NONZERO TEMPERATURES}

Following Feynman, the Yang-Mills (YM) partition function can be written as a functional integral over the YM 4-potentials $A_{\mu}(t, \mathbf{x})$ that are traceless hermitian $N \times N$ matrices, satisfying periodic boundary conditions in imaginary (or Euclidean) time:

$$
\begin{array}{r}
\mathscr{Z}=\int D A_{\mu}(t, \mathbf{x}) \exp \left(-\frac{1}{2 g^{2}} \int_{0}^{\frac{1}{T}} d t \int d^{3} \mathbf{x} \operatorname{Tr} F_{\mu v} F_{\mu v}\right), \\
A_{\mu}\left(t+\frac{1}{T}, \mathbf{x}\right)=A_{\mu}(t, \mathbf{x}), T=\text { temperature }
\end{array}
$$

where $F_{\mu \nu}=\partial_{\mu} A_{v}-\partial_{v} A_{\mu}-i\left[A_{\mu} A_{v}\right]$ is the YM field strength.

An important variable is the Polyakov loop; it is the path-ordered exponent in time direction, hence it can depend only on the space point $\mathbf{x}$ :

$$
L(\mathbf{x})=\mathscr{P} \exp \left(i \int_{0}^{\frac{1}{T}} d t A_{4}(t, \mathbf{x})\right), \quad L \in S U(N) .
$$

Under space-dependent gauge transformations it transforms as $L \rightarrow U^{-1} L U$. The eigenvalues of $L(\mathbf{x})$ are gauge invariant; we parameterize them as

$$
L=\operatorname{diag}\left(e^{2 \pi i \mu_{1}}, e^{2 \pi i \mu_{2}}, \ldots, e^{2 \pi i \mu_{N}}\right),
$$

$\mu_{1}+\ldots+\mu_{N}=0$, and assume that the phases of these eigenvalues are ordered: $\mu_{1} \leq \mu_{2} \leq \ldots \leq \mu_{N} \leq \mu_{N+1} \equiv$ 


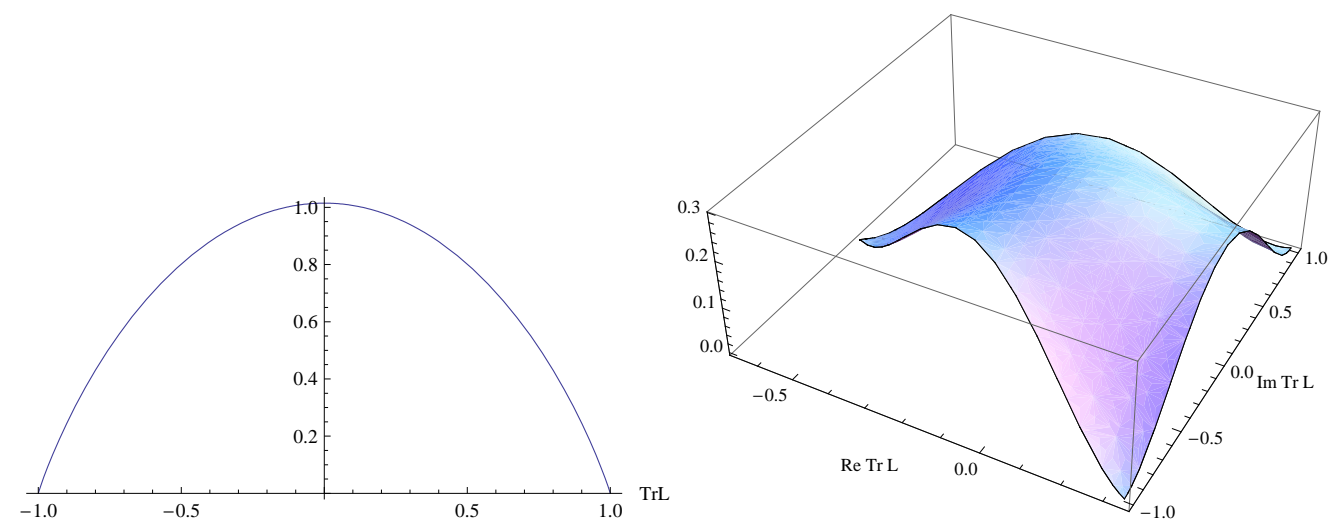

FIGURE 1. The perturbative potential energy as function of the Polyakov line for the $S U(2)$ (left) and $S U(3)$ (right) groups. It has minima where the Polyakov loop is one of the $N$ elements of the center $Z_{N}$ and is maximal at the "confining" holonomy.

$\mu_{1}+1$. We shall call the set of $N$ phases $\left\{\mu_{m}\right\}$ the "holonomy" for short. Apparently, shifting $\mu$ 's by integers does not change the eigenvalues, hence all quantities have to be periodic in all $\mu$ 's with a period equal to unity.

The holonomy is said to be "trivial" if $L$ belongs to one of the $N$ elements of the group center $Z_{N}$. For example, in $S U(3)$ the three trivial holonomies are

$$
\begin{aligned}
& \mu_{1}=\mu_{2}=\mu_{3}=0 \quad \Longrightarrow \quad L=\mathbf{1}_{3}, \\
& \mu_{1}=-\frac{2}{3}, \mu_{2}=\frac{1}{3}, \mu_{3}=\frac{1}{3} \quad \Longrightarrow \quad L=e^{\frac{2 \pi i}{3}} \mathbf{1}_{3}, \\
& \mu_{1}=-\frac{1}{3}, \mu_{2}=-\frac{1}{3}, \mu_{3}=\frac{2}{3} \quad \Longrightarrow \quad L=e^{-\frac{2 \pi i}{3}} \mathbf{1}_{3} .
\end{aligned}
$$

Trivial holonomy corresponds to equal $\mu$ 's, modulo unity. Out of all possible combinations of $\mu$ 's a distinguished role is played by equidistant $\mu$ 's corresponding to $\operatorname{Tr} L=0$ :

$$
\mu_{m}^{\mathrm{conf}}=-\frac{1}{2}-\frac{1}{2 N}+\frac{m}{N}
$$

For example, in $S U(3)$ it is

$$
\mu_{1}=-\frac{1}{3}, \mu_{2}=0, \mu_{3}=\frac{1}{3} \Longrightarrow L=\operatorname{diag}\left(e^{-\frac{2 \pi i}{3}}, 1, e^{\frac{2 \pi i}{3}}\right) \text {. }
$$

We shall call it "most non-trivial" or "confining" holonomy as it corresponds to $\operatorname{Tr} L=0$ which is the $1^{\text {st }}$ confinement requirement.

Immediately, an interesting question arises: Imagine we take the YM partition function (2) and integrate out all degrees of freedom except the eigenvalues $\left\{\mu_{m}\right\}$ of the Polyakov loop $L(\mathbf{x})$ which, in addition, we take slowly varying in space. What is the effective action for $\mu$ 's? What set of $\mu$ 's is preferred dynamically by the YM system of fields?

First of all, one can address this question in perturbation theory: the result for the potential energy as function of $\mu$ 's is [3, 4]

$$
P^{\text {pert }}=\left.\frac{(2 \pi)^{2} T^{3}}{3} \sum_{m>n}^{N}\left(\mu_{m}-\mu_{n}\right)^{2}\left[1-\left(\mu_{m}-\mu_{n}\right)\right]^{2}\right|_{\bmod 1} .
$$

It is proportional to $T^{3}$ (by dimensions) and has exactly $N$ zero minima when all $\mu$ 's are equal modulo unity, see Fig. 1. Hence, $P^{\text {pert }}$ says that at least at high temperatures the system prefers one of the $N$ trivial holonomies corresponding to the Polyakov loop being one of the $N$ elements of the center $Z_{N}$. However, terms with gradients of $\mu$ 's in the effective action become negative near "trivial" holonomy, signalling its instability even in perturbation theory [5].

It is interesting that in the supersymmetric $\mathscr{N}=1$ version of the YM theory (where in addition to gluons there are spin- $\frac{1}{2}$ gluinos in the adjoint representation) the perturbative potential energy (7) is absent in all orders owing to fermion-boson cancelation, but the nonperturbative potential energy is nonzero. Moreover, it is known exactly as function of $\mu$ 's [6]: it has a single minimum at precisely the "most non-trivial" or "confining" holonomy (5). The result can be traced to the semiclassical contribution of dyons, which turns out to be exact owing to supersymmetry.

In the non-supersymmetric pure YM theory, the dyoninduced contribution cannot be computed exactly but only in the semiclassical approximation (this is what the paper is about), and the perturbative contribution (7) is present, too. We shall show below that a semiclassical configuration - an ensemble of dyons with quantum fluctuations about it - generates a nonperturbative free energy shown in Fig. 2. It has the opposite behavior of the perturbative one, having the minimum at the equidistant (confining) values of the $\mu$ 's. There is a fight between the perturbative and nonperturbative contributions to the free energy [7]. Since the perturbative contribution to the free energy is $\sim T^{4}$ with respect to the nonperturbative one, it 


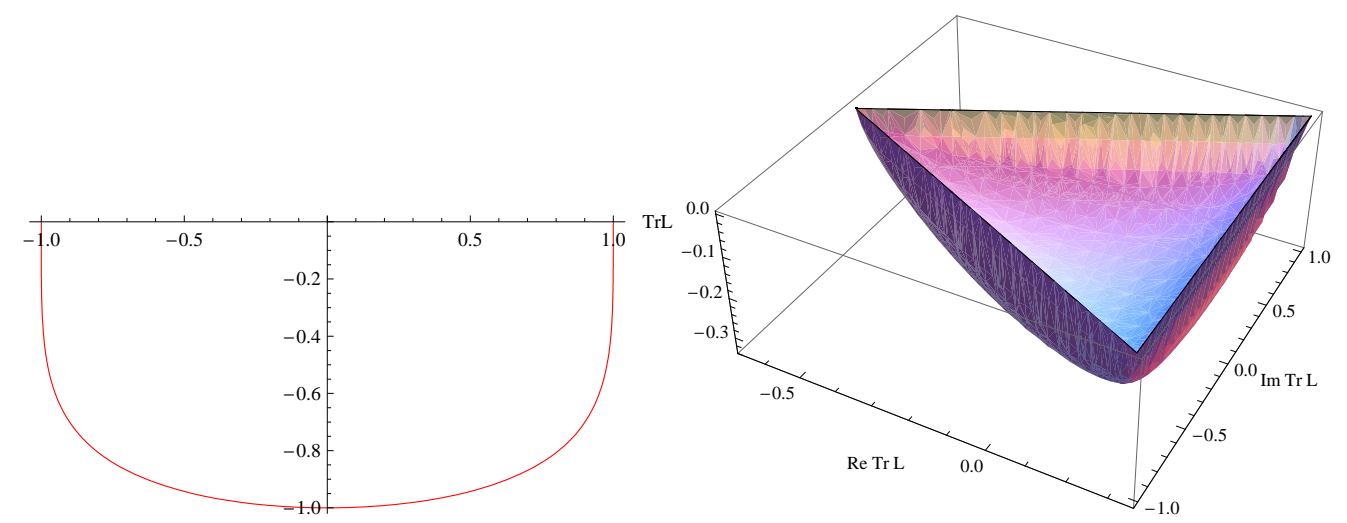

FIGURE 2. The dyon-induced nonperturbative potential energy as function of the Polyakov line for the $S U(2)(l e f t)$ and $S U(3)$ (right) groups. Contrary to the perturbative potential energy, it has a single and non-degenerate minimum at the confining holonomy corresponding to $\operatorname{Tr} L=0$.

certainly wins when temperatures are high enough, and the system is then forced into one of the $N$ vacua thus breaking spontaneously the $Z_{N}$ symmetry. At low temperatures the nonperturbative contribution prevails forcing the system into the confining vacuum. At a critical $T_{c}$ there is a confinement-deconfinement phase transition. It turns out to be of the second order for $N=2$ but first order for $N=3$ and higher, in agreement with lattice findings.

\section{DYON SADDLE POINTS}

Dyons or Bogomolny-Prasad-Sommerfield (BPS) monopoles [8] are (anti) self-dual solutions of the nonlinear Maxwell equations, $D_{\mu}^{a b} F_{\mu v}^{b}=0$. In $S U(N)$ there are exactly $N$ kinds of 'fundamental' dyons with Coulomb asymptotics for both electric and magnetic fields (hence the term "dyon"):

$$
\pm \mathbf{E}=\mathbf{B} \stackrel{|\mathbf{x}| \rightarrow \infty}{=} \frac{1}{2} \frac{\mathbf{x}}{|\mathbf{x}|^{3}} \times\left\{\begin{array}{c}
\operatorname{diag}(1,-1,0, \ldots, 0,0) \\
\operatorname{diag}(0,1,-1, \ldots, 0,0) \\
\ldots \\
\operatorname{diag}(0,0,0, \ldots, 1,-1) \\
\operatorname{diag}(-1,0,0, \ldots, 0,1)
\end{array} .\right.
$$

Dyon solutions are labeled by the holonomy or the set of $\mu$ 's at spatial infinity:

$$
A_{4}(|\mathbf{x}| \rightarrow \infty) \rightarrow 2 \pi T \operatorname{diag}\left(\mu_{1}, \mu_{2}, \ldots, \mu_{N}\right) .
$$

The explicit expressions for the solutions in various gauges can be found e.g. in the Appendix of Ref. [9]. Inside the cores which are of the size $\sim 1 /\left(T v_{m}\right)$, the fields are large, nonlinearity is essential. The action density is time-independent everywhere and is proportional to the temperature. Isolated dyons are thus $3 d$ objects but with finite action independent of temperature:

$$
S_{\text {dyon }}=\frac{2 \pi}{\alpha_{s}} v_{m}, \quad v_{m} \equiv \mu_{m+1}-\mu_{m}, \quad \sum_{m} v_{m}=1,
$$

(here $\mu_{N+1} \equiv \mu_{1}+1$ ). The full action of all $N$ kinds of well-separated dyons together is that of one standard instanton: $S_{\text {inst }}=2 \pi / \alpha_{s}$.

In the semiclassical approach, one has first of all to find the statistical weight with which a given classical configuration enters the partition function. It is given by $\exp (-$ Action $)$, times the determinant ${ }^{-1 / 2}$ from small quantum oscillations about the saddle point. For an isolated dyon as a saddle-point configuration, this factor diverges linearly in the infrared region owing to the slow Coulomb decrease of the dyon field (8). It means that isolated dyons are not acceptable as saddle points: they have zero weight, despite finite classical action. However, one may look for classical solutions that are superpositions of $N$ fundamental dyons, with zero net magnetic charge. The small-oscillation determinant must be infrared-finite for such classical solutions, if they exist.

\section{INSTANTONS WITH NON-TRIVIAL HOLONOMY}

The needed classical solution has been found a decade ago by Kraan and van Baal [10] and independently by Lee and Lu [11], see also [12]. We shall call them for short the "KvBLL instantons"; an alternative name is "calorons with nontrivial holonomy". The solution was first found for the $S U(2)$ group but soon generalized to an arbitrary $S U(N)$ [13], see [14] for a review.

The general solution $A_{\mu}^{\mathrm{KvBLL}}$ depends on Euclidean time $t$ and space $\mathbf{x}$ and is parameterized by $3 N$ positions of $N$ kinds of 'constituent' dyons in space $\mathbf{x}_{1}, \ldots, \mathbf{x}_{N}$ and their $U(1)$ phases $\psi_{1}, \ldots, \psi_{N}$. All in all, there are $4 N$ collective coordinates characterizing the solution (called the moduli space), of which the action $S_{\text {inst }}=2 \pi / \alpha_{s}$ is in fact independent, as it should be for a general solution with a unity topological charge. The solution 

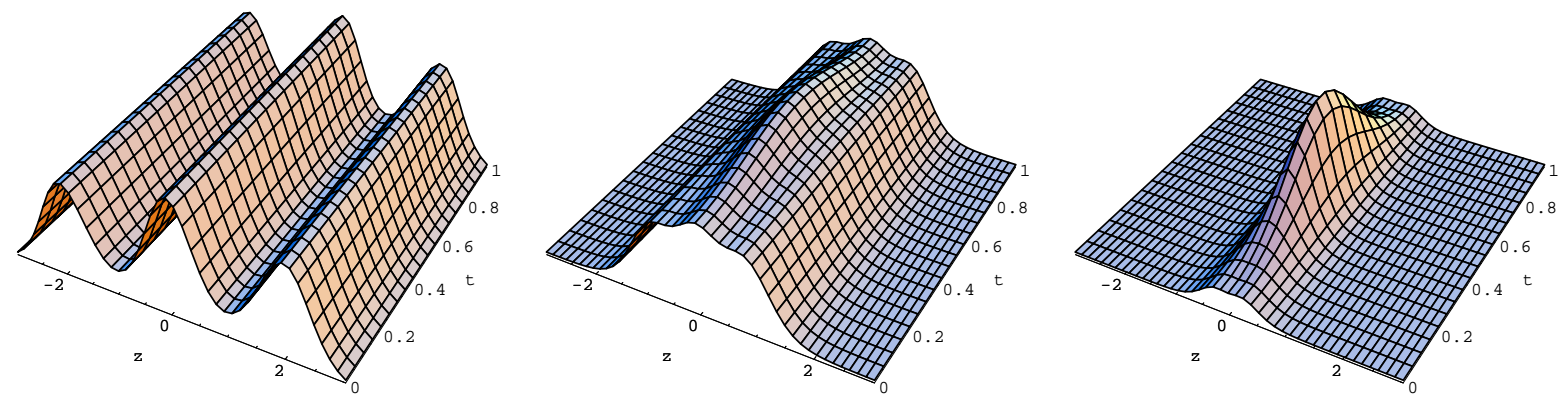

FIGURE 3. Action density inside the $S U(3) \mathrm{KvBLL}$ instanton as function of time and one space coordinate, for large (left), intermediate (middle) and small (right) separations between the three constituent dyons.

also depends explicitly on temperature $T$ and on the holonomy $\mu_{1}, \ldots, \mu_{N}$ :

$A_{\mu}^{\mathrm{KvBLL}}=\bar{A}_{\mu}^{a}\left(t, \mathbf{x} ; \mathbf{x}_{1}, \ldots, \mathbf{x}_{N}, \psi_{1}, \ldots, \psi_{N} ; T, \mu_{1}, \ldots, \mu_{N}\right)$.

The solution is a relatively simple expression given by elementary functions. If the holonomy is trivial (all $\mu$ 's are equal modulo unity) the expression takes the form of the strictly periodic $O(3)$ symmetric caloron [15] reducing further to the standard $O(4)$ symmetric BPST instanton [16] in the $T \rightarrow 0$ limit. At small temperatures but arbitrary holonomy, the KvBLL instanton also has only a small $\mathscr{O}(T)$ difference with the standard instanton.

One can plot the action density of the KvBLL instanton in various corners of the parameter (moduli) space, see Fig. 3.

When all dyons are far apart one observes $N$ static (i.e. time-independent) objects, the isolated dyons. As they merge, the configuration is not static anymore, it becomes a process in time. In the limiting case of a complete merger, the configuration becomes a $4 d$ lump resembling the standard instanton. The full (integrated) action is exactly the same $S_{\text {inst }}=2 \pi / \alpha_{s}$ for any choice of the dyon separations. It means that classically dyons do not interact. However, they do experience a peculiar interaction at the quantum level to which we proceed.

\section{QUANTUM WEIGHT OF MANY DYONS}

Remarkably, the small-oscillation determinant about a single KvBLL $S U(N)$ instanton made of $N$ differentkind dyons can be computed exactly [17, 18]. With this experience, the quantum weight of an arbitrary number of dyons of $N$ kinds has been suggested in Ref. [2]. In the YM partition function, there are saddle points corresponding to any set of $K_{m}$ dyons. In the thermodynamic limit $V \rightarrow \infty$ one needs to take a saddle point with $\mathscr{O}(V)$ dyons. Let $K_{m}$ be the number of dyons of kind $m(m=1 \ldots N)$ and let $\mathbf{x}_{m i}$ be the coordinate of the $i^{\text {th }}$ dyon of kind $m\left(i=1 \ldots K_{m}\right)$. In the semiclassical approximation the YM partition function is approximated by the partition function of a grand canonical ensemble of $K_{1}+K_{2}+\ldots+K_{N}$ dyons,

$$
\mathscr{Z}=\sum_{K_{1} \ldots K_{N}} \frac{1}{K_{1} ! \ldots K_{N} !} \prod_{m=1}^{N} \prod_{i=1}^{K_{m}} \int\left(d \mathbf{x}_{m i} f\right) \sqrt{\operatorname{det} g\left(\mathbf{x}_{m i}\right)},
$$

where $g\left(\mathbf{x}_{m i}\right)$ is a $4\left(K_{1}+\ldots+K_{N}\right) \times 4\left(K_{1}+\ldots+K_{N}\right)$ metric tensor of the dyons' moduli space, composed by the overlaps of zero modes of individual dyons, and $f$ is the fugacity,

$$
f=\frac{N^{2}}{16 \pi^{3} \lambda^{2}} \frac{\Lambda^{4}}{T}=\mathscr{O}\left(N^{2}\right) .
$$

The bare 't Hooft coupling constant $\lambda$ is renormalized and starts to "run" only at the 2-loop level not considered here. Eventually, its argument will be the largest scale in the problem, be it the temperature or the equilibrium density of dyons.

It is not difficult to find the metric tensor $g\left(\mathbf{x}_{m i}\right)$ for well-separated dyons. In this case the four zero modes $\phi_{\mu}^{(\kappa)}(\kappa=1,2,3,4)$ of individual dyons are given by the components of the field strength: $\phi_{\mu}^{(\kappa)}=F_{\mu \kappa}$. The zero modes for the $m^{\text {th }}$ kind of dyon are normalized to its action, $\int \operatorname{Tr} \phi_{\mu}^{(\kappa)} \phi_{\mu}^{(\lambda)} \sim \delta^{\kappa \lambda} v_{m}$ (see Eq. (10) ) and hence depend on the holonomy. Since the field strengths decay as $1 / r^{2}$ (see Eq. (8)) the overlaps between zero modes are Coulomb-like, and only those that are nearest neighbors in $m$ do interact. In fact, the diagonal components of the metric tensor also acquire Coulomb-like corrections since the action of individual dyons is actually normalized to its asymptotic field $A_{4}$ that gets Coulomb corrections from other dyons.

As a result, we obtain the $4\left(K_{1}+\ldots+K_{N}\right) \times 4\left(K_{1}+\right.$ $\left.\ldots+K_{N}\right)$ metric tensor $g\left(\mathbf{x}_{m i}\right)$ with Coulomb interactions as entries, and the $v_{m}$ 's on the diagonal. It turns out that its determinant is a square of the determinant of a related 
matrix, $\sqrt{\operatorname{det} g}=\operatorname{det} G$ where $G$ is a $\left(K_{1}+\ldots+K_{N}\right) \times$ $\left(K_{1}+\ldots+K_{N}\right)$ matrix:

$$
\begin{gathered}
G_{m i, n j}=\delta_{m n} \delta_{i j}\left(4 \pi v_{m}+\sum_{k} \frac{1}{T\left|\mathbf{x}_{m i}-\mathbf{x}_{m-1, k}\right|}\right. \\
\left.+\sum_{k} \frac{1}{T\left|\mathbf{x}_{m i}-\mathbf{x}_{m+1, k}\right|}-2 \sum_{k \neq i} \frac{1}{T\left|\mathbf{x}_{m i}-\mathbf{x}_{m k}\right|}\right) \\
-\frac{\delta_{m, n-1}}{T\left|\mathbf{x}_{m i}-\mathbf{x}_{m+1, j}\right|}-\frac{\delta_{m, n+1}}{T\left|\mathbf{x}_{m i}-\mathbf{x}_{m-1, j}\right|}+\left.2 \frac{\delta_{m n}}{T\left|\mathbf{x}_{m i}-\mathbf{x}_{m j}\right|}\right|_{i \neq j},
\end{gathered}
$$

where $\mathbf{x}_{m i}$ is the coordinate of the $i^{\text {th }}$ dyon of kind $m$. The matrix $G$ has the following nice properties:

- symmetry: $G_{m i, n j}=G_{n j, m i}$

- overall "neutrality": the sum of Coulomb interactions in non-diagonal entries cancel those on the diagonal: $\sum_{n j} G_{m i, n j}=4 \pi v_{m}$

- identity loss: dyons of the same kind are indistinguishable, meaning mathematically that $\operatorname{det} G$ is symmetric under permutation of any pair of dyons $(i \leftrightarrow j)$ of the same kind $m$. Dyons do not 'know' to which instanton they belong to

- attraction/repulsion: if one decreases the separation between same-kind dyons or increases the separation between different-kind dyons, the $\operatorname{det} G$ decreases. It means that same-kind dyons repulse each other whereas different-kind dyons attract each other. The $\operatorname{det} G$ measure favors formation of neutral clusters with $N$ different kinds of dyons

- factorization: in the geometry when dyons fall into $K$ well separated neutral clusters of $N$ dyons of different kinds, $\operatorname{det} G$ factorizes into a product of exact integration measures for $K$ KvBLL instantons [19, 20] valid for any separations between different-kind dyons, including their strong overlap

- last but not least, the metric $g$ corresponding to $G$ is hyper-Kähler, as it should be for the moduli space of a self-dual classical field [21]. In fact, it is a severe restriction on the metric.

An overall constant factor depending on the holonomy and temperature, $\exp \left(-P^{\text {pert }} V\right)$, is understood in Eq. (12), where $P^{\text {pert }}$ is the perturbative gluon loop (7) in the background of a constant field $A_{4}(9)$. This factor arises from the non-zero modes in the fluctuation determinant about dyons and is necessarily present as most of the $3 d$ space outside the dyons' core is just a constant $A_{4}$ background. Indeed the calculation [17, 18] exhibits this factor which is the only one proportional to the 3 -volume $V$.

The ensemble defined by a determinant of a matrix whose dimension is the number of particles, is not a usual one. More customary, the interaction is given by the Boltzmann factor $\exp \left(-U_{\text {int }}\left(\mathbf{x}_{1}, \ldots\right)\right)$. Of course, one can always present the determinant in that way using the identity $\operatorname{det} G=\exp (\operatorname{Tr} \log G) \equiv \exp \left(-U_{\text {int }}\right)$ but the interactions will then include three-, four-, five-... body forces. At the same time, it is precisely the determinant form of the interaction that makes the statistical physics of dyons an exactly solvable problem.

\section{STATISTICAL PHYSICS OF DYONS AS A QUANTUM FIELD THEORY}

It is possible to present the grand canonical ensemble of dyons, governed by the interaction (14) as an equivalent $3 d$ quantum field theory. This will enable us to compute various correlation functions of interest.

To proceed to the quantum field theory description we use two mathematical tricks.

1. "Fermionization" (Berezin [22]). It is helpful to exponentiate the Coulomb interactions rather than keeping them in $\operatorname{det} G$. To that end one presents the determinant of a matrix as an integral over a finite number of anticommuting Grassmann variables $\left(\left\{\psi_{A}^{\dagger} \psi_{B}\right\}=\delta_{A B}\right)$ :

$$
\operatorname{det}\left(G_{A B}\right)=\int \prod_{A} d \psi_{A}^{\dagger} d \psi_{A} \exp \left(\psi_{A}^{\dagger} G_{A B} \psi_{B}\right) .
$$

Now we have the two-body Coulomb interactions in the exponent and it is possible to use the second trick.

2. "Bosonization" (Polyakov [23]). One can present the Coulomb interactions in the exponent with the help of a Gaussian integral over an auxiliary field $\phi$ :

$$
\begin{aligned}
& \exp \left(\sum_{m, n} \frac{Q_{m} Q_{n}}{\left|\mathbf{x}_{m}-\mathbf{x}_{n}\right|}\right)=\int D \phi \exp \left[-\int d \mathbf{x}\right. \\
& \left.\cdot\left(\frac{1}{16 \pi} \partial_{i} \phi \partial_{i} \phi+\rho \phi\right)\right]=\exp \left(\int \rho \frac{4 \pi}{\triangle} \rho\right), \\
& \rho=\sum Q_{m} \delta\left(\mathbf{x}-\mathbf{x}_{m}\right) .
\end{aligned}
$$

After applying the first trick the "charges" $Q_{m}$ become Grassmann variables but after applying the second one, it becomes easy to integrate them out since the square of a Grassmann variable is zero. In fact one needs $2 N$ boson fields $\mathrm{v}_{m}, \mathrm{w}_{m}$ to reproduce diagonal elements of $G$ and $2 N$ anticommuting ("ghost") fields $\chi_{m}^{\dagger}, \chi_{m}$ to present the non-diagonal elements. The chain of identities is accomplished in Ref. [2] and the result for the partition function for the dyon ensemble (12) is, identically, a path integral defining a quantum field theory in 3 dimensions:

$$
\mathscr{Z}=\int D \chi^{\dagger} D \chi D \mathrm{v} D \mathrm{w} \exp \int d^{3} x\left\{\frac { T } { 4 \pi } \left(\partial_{i} \chi_{m}^{\dagger} \partial_{i} \chi_{m}\right.\right.
$$




$$
\begin{aligned}
& \left.+\partial_{i} \mathrm{v}_{m} \partial_{i} \mathrm{w}_{m}\right)+f\left[\left(-4 \pi \mu_{m}+\mathrm{v}_{m}\right) \frac{\partial \mathscr{F}}{\partial \mathrm{w}_{m}}\right. \\
& \left.\left.+\chi_{m}^{\dagger} \frac{\partial^{2} \mathscr{F}}{\partial \mathrm{w}_{m} \partial \mathrm{w}_{n}} \chi_{n}\right]\right\}, \quad \mathscr{F}=\sum_{m=1}^{N} e^{\mathrm{w}_{m}-\mathrm{w}_{m+1}}
\end{aligned}
$$

The fields $\mathrm{v}_{m}$ have the meaning of the asymptotic Abelian electric potentials of dyons,

$$
\begin{aligned}
\left(A_{4}\right)_{m n} & =\delta_{m n} A_{m 4}, \\
A_{m 4}(\mathbf{x}) / T & =2 \pi \mu_{m}-\frac{1}{2} \mathrm{v}_{m}(\mathbf{x}), \quad \mathbf{E}_{m}=\nabla A_{m 4},
\end{aligned}
$$

while $\mathrm{w}_{m}$ have the meaning of the dual (or magnetic) Abelian potentials. Note that the kinetic energy for the $\mathrm{v}_{m}, \mathrm{w}_{m}$ fields has only the mixing term $\partial_{i} \mathrm{v}_{m} \partial_{i} \mathrm{w}_{m}$ which is nothing but the Abelian duality transformation $\mathbf{E} \cdot \mathbf{B}$. The function $\mathscr{F}(w)$ in $(15)$ where one assumes a cyclic summation over $m$, is known as the periodic (or affine) Toda lattice.

Although the Lagrangian in Eq. (15) describes a highly nonlinear interacting quantum field theory, it is in fact exactly solvable! To prove it, one observes that the fields $\mathbf{v}_{m}$ enter the Lagrangian only linearly, therefore one can integrate them out. It leads to a functional $\delta$-function:

$$
\int D \mathrm{v}_{m} \longrightarrow \delta\left(-\frac{T}{4 \pi} \partial^{2} \mathrm{w}_{m}+f \frac{\partial \mathscr{F}}{\partial \mathrm{w}_{m}}\right)
$$

This $\delta$-function restricts possible fields $\mathrm{w}_{m}$ over which one still has to integrate in eq. (15). Let $\overline{\mathrm{w}}_{m}$ be a solution to the argument of the $\delta$-function. Integrating over small fluctuations about $\overline{\mathrm{w}}$ gives the Jacobian

$$
\mathrm{Jac}=\operatorname{det}^{-1}\left(-\frac{T}{4 \pi} \partial^{2} \delta_{m n}+\left.f \frac{\partial^{2} \mathscr{F}}{\partial \mathrm{w}_{m} \partial \mathrm{w}_{n}}\right|_{\mathrm{w}=\overline{\mathrm{w}}}\right) .
$$

Remarkably, exactly the same functional determinant but in the numerator arises from integrating over the ghost fields, for any background $\overline{\mathrm{w}}$. Therefore, all quantum corrections cancel exactly between the boson and ghost fields (a characteristic feature of supersymmetry), and the ensemble of dyons is basically governed by a classical field theory.

To find the ground state we examine the fields' potential energy being $-4 \pi f \mu_{m} \partial \mathscr{F} / \partial \mathrm{w}_{m}$ which we prefer to write restoring $v_{m}=\mu_{m+1}-\mu_{m}$ and $\mathscr{F}$ as

$$
\mathscr{P}=-4 \pi f V \sum_{m} v_{m} e^{\mathrm{w}_{m}-\mathrm{w}_{m+1}}
$$

(the volume factor arises for constant fields $\mathrm{w}_{m}$ ). One has first to find the stationary point in $\mathrm{w}_{m}$ for a given set of $v_{m}$ 's. It leads to the equations

$$
\frac{\partial \mathscr{P}}{\partial \mathrm{w}_{m}}=0
$$

whose solution is

$$
e^{w_{1}-w_{2}}=\frac{\left(v_{1} v_{2} v_{3} \ldots v_{N}\right)^{\frac{1}{N}}}{v_{1}}, \quad \text { etc. }
$$

Putting it back into eq. (19) we obtain

$$
\mathscr{P}=-4 \pi f V N\left(v_{1} v_{2} \ldots v_{N}\right)^{\frac{1}{N}}, \quad v_{1}+\ldots+v_{N}=1 .
$$

The minimum equal $\mathscr{P}_{\min }=-4 \pi f V$ is achieved at $v_{1}=$ $\ldots=v_{N}=\frac{1}{N}$, that is at equidistant, confining value of the holonomy, cf. Eq. (5). We have also proven that the result is exact, as all potential quantum corrections cancel in the partition function (15).

Given this cancelation, the key finding - that the dyoninduced free energy has the minimum at the confining value of holonomy - is trivial. If all Coulomb interactions cancel after integration over dyons' positions, the weight of a many-dyon configuration is the same as if they were infinitely dilute (although they are not). Then the weight, what concerns the holonomy, is proportional to the product of diagonal matrix elements of $G$ in the dilute limit, that is to the normalization integrals for dyon zero modes, that is to the product of the dyon actions $\sim v_{m}$ where $v_{m}=\mu_{m+1}-\mu_{m}$ and $v_{N}=\mu_{1}+1-\mu_{N}$ such that $v_{1}+v_{2}+\ldots+v_{N}=1$. The sum of all $N$ kinds of dyons' actions is fixed and equal to the instanton action, however, it is the product of actions that defines the weight. The product is maximal when all actions are equal, hence the equidistant or confining $\mu$ 's are statistically preferred. Thus, the average Polyakov line is zero, $<\operatorname{Tr} L>=0$.

\section{HEAVY QUARK POTENTIAL}

The field-theoretic representation of the dyon ensemble enables one to compute various YM correlation functions in the semiclassical approximation. The key observables relevant to confinement are the correlation function of two Polyakov lines (defining the heavy quark potential), and the average of large Wilson loops. A detailed calculation of these quantities is performed in Ref. [2]; here we only present the results and discuss the meaning.

\section{$N$-ality and $k$-strings}

From the viewpoint of confinement, all irreducible representations of the $S U(N)$ group fall into $N$ classes: those that appear in the direct product of any number of adjoint representations, and those that appear in the direct product of any number of adjoint representations with the irreducible representation being the rank- $k$ antisymmetric tensor, $k=1, \ldots, N-1$. " $N$-ality" is said to be 
zero in the first case and equal to $k$ in the second. $N$-alityzero representations transform trivially under the center of the group $Z_{N}$; the rest acquire a phase $2 \pi k / N$.

One expects that there is no asymptotic linear potential between static color sources in the adjoint representation as such sources are screened by gluons. If a representation is found in a direct product of some number of adjoint representations and a rank- $k$ antisymmetric representation, the adjoint ones "cancel out" as they can be all screened by an appropriate number of gluons. Therefore, from the confinement viewpoint all $N$-ality $=k$ representations are equivalent and there are only $N-1$ string tensions $\sigma_{k, N}$ being the coefficients in the asymptotic linear potential for sources in the antisymmetric rank- $k$ representation. They are called " $k$-strings".

The value $k=1$ corresponds to the fundamental representation whereas $k=N-1$ corresponds to the representation conjugate to the fundamental [quarks and antiquarks]. In general, the rank- $(N-k)$ antisymmetric representation is conjugate to the rank- $k$ one; it has the same dimension and the same string tension, $\sigma_{k, N}=\sigma_{N-k, N}$.

The behavior of $\sigma_{k, N}$ as function of $k$ and $N$ is an important issue as it discriminates between various confinement mechanisms. On general $N$-counting grounds one can only infer that at large $N$ and $k \ll$ $N, \sigma_{k, N} / \sigma_{1, N}=(k / N)\left(1+\mathscr{O}\left(1 / N^{2}\right)\right)$. Important, there should be no $\mathscr{O}\left(N^{-1}\right)$ correction [24]. A popular version called "Casimir scaling", according to which the string tension is proportional to the Casimir operator for a given representation (it stems from an idea that confinement is somehow related to the modification of a one-gluon exchange at large distances), does not satisfy this restriction.

\section{Correlation function of Polyakov lines}

To find the potential energy $V_{k, N}$ of static "quark" and "antiquark" transforming according to the antisymmetric rank- $k$ representation, one has to consider the correlation of Polyakov lines in the appropriate representation:

$$
\left\langle\operatorname{Tr} L_{k, N}\left(\mathbf{z}_{1}\right) \operatorname{Tr} L_{k, N}^{\dagger}\left(\mathbf{z}_{2}\right)\right\rangle=\text { const. } \exp \left(-\frac{V_{k, N}\left(\mathbf{z}_{1}-\mathbf{z}_{2}\right)}{T}\right) .
$$

Far away from dyons' cores the field is Abelian and in the field-theoretic language of Eq. (15) is given by Eq. (16). Therefore, the Polyakov line in the fundamental representation is

$$
\operatorname{Tr} L(\mathbf{z})=\sum_{m=1}^{N} Z_{m}, Z_{m}=\exp \left(2 \pi i \mu_{m}-\frac{i}{2} \mathrm{v}_{m}(\mathbf{z})\right)
$$

In the general antisymmetric rank- $k$ representation

$$
\operatorname{Tr} L_{k, N}(\mathbf{z})=\sum_{m_{1}<m_{2}<\ldots<m_{k}}^{N} Z_{m_{1}} Z_{m_{2}} \ldots Z_{m_{k}}
$$

where cyclic summation from 1 to $N$ is assumed.

The average (22) can be computed from the quantum field theory (15). Inserting the two Polyakov lines (24) into Eq. (15) we observe that the Abelian electric potential $\mathrm{v}_{m}$ enters linearly in the exponent as before. Therefore, it can be integrated out, leading to a $\delta$-function for the dual field $\mathrm{w}_{m}$, which is now shifted by the source (cf. Eq. (17)):

$$
\begin{aligned}
\int D \mathrm{v}_{m} & \longrightarrow \prod_{m} \delta\left(-\frac{T}{4 \pi} \partial^{2} \mathrm{w}_{m}+f \frac{\partial \mathscr{F}}{\partial \mathrm{w}_{m}}\right. \\
& -\frac{i}{2} \delta\left(\mathbf{x}-\mathbf{z}_{\mathbf{1}}\right)\left(\delta_{m m_{1}}+\ldots+\delta_{m m_{k}}\right) \\
& \left.+\frac{i}{2} \delta\left(\mathbf{x}-\mathbf{z}_{\mathbf{2}}\right)\left(\delta_{m n_{1}}+\ldots+\delta_{m n_{k}}\right)\right) .
\end{aligned}
$$

One has to find the dual field $\mathrm{w}_{m}(\mathbf{x})$ nullifying the argument of this $\delta$-function, plug it into the action

$$
\exp \left(\int d \mathbf{x} \frac{4 \pi f}{N} \mathscr{F}(w)\right)
$$

and sum over all sets $\left\{m_{1}<m_{2}<\ldots<\right.$ $\left.m_{k}\right\}, \quad\left\{n_{1}<n_{2}<\ldots<n_{k}\right\} \quad$ with the weight $\exp \left(2 \pi i\left(m_{1}+\ldots+m_{k}-n_{1}-\ldots-n_{k}\right) / N\right)$. The Jacobian from resolving the $\delta$-function again cancels exactly with the determinant arising from ghosts. Therefore, the calculation of the correlator (22), sketched above, is exact.

At large separations between the sources $\left|\mathbf{z}_{1}-\mathbf{z}_{2}\right|$, the fields $\mathrm{w}_{m}$ resolving the $\delta$-function are small and one can expand the Toda chain:

$$
\mathscr{F}(w)=\sum_{m} e^{\mathrm{w}_{m}-\mathrm{w}_{m+1}} \approx N+\frac{1}{2} \mathrm{w}_{m} \mathscr{M}_{m n} \mathrm{w}_{n},
$$

where

$$
\mathscr{M}=\left(\begin{array}{cccccc}
2 & -1 & 0 & \ldots & 0 & -1 \\
-1 & 2 & -1 & \ldots & 0 & 0 \\
0 & -1 & 2 & -1 & \ldots & 0 \\
\ldots & \ldots & \ldots & \ldots & \ldots & \ldots \\
-1 & 0 & 0 & \ldots & -1 & 2
\end{array}\right)
$$

As apparent from Eq. (26), the eigenvalues of $\mathscr{M}$ determine the spectrum of the dual fields $\mathrm{w}_{m}$. There is one zero eigenvalue which decouples from everywhere, and $N-1$ nonzero eigenvalues

$$
\mathscr{M}^{(k)}=\left(2 \sin \frac{\pi k}{N}\right)^{2}, \quad k=1, \ldots, N-1 .
$$


Certain orthogonality relation imposes the selection rule: the asymptotics of the correlation function of two Polyakov lines in the antisymmetric rank- $k$ representation is determined by precisely the $k^{\text {th }}$ eigenvalue. We obtain [2]

$$
\begin{aligned}
& \left\langle\operatorname{Tr} L_{k, N}\left(\mathbf{z}_{1}\right) \operatorname{Tr} L_{k, N}^{\dagger}\left(\mathbf{z}_{2}\right)\right\rangle \\
& \stackrel{z_{12} \rightarrow \infty}{=} \text { const. } \exp \left(-\left|\mathbf{z}_{1}-\mathbf{z}_{2}\right| M \sqrt{\mathscr{M}^{(k)}}\right)
\end{aligned}
$$

where $M$ is the 'dual photon' mass,

$$
M=\sqrt{\frac{4 \pi f}{T}}=\frac{N \Lambda^{2}}{2 \pi \lambda T}=\mathscr{O}(N) .
$$

Comparing it with the definition of the heavy quark potential (22) we find that there is an asymptotically linear potential between static "quarks" in any $N$-ality nonzero representation, with the $k$-string tension

$$
\sigma_{k, N}=M T \sqrt{\mathscr{M}^{(k)}}=2 M T \sin \frac{\pi k}{N}=\frac{\Lambda^{2}}{\lambda} \frac{N}{\pi} \sin \frac{\pi k}{N} .
$$

This is the so-called 'sine regime': it has been found before in certain supersymmetric theories [25]. Lattice simulations [26] support this regime, whereas another lattice study [27] gives somewhat smaller values but within two standard deviations from the values following from eq. (31).

We see that at large $N$ and $k \ll N, \sigma_{k, N} / \sigma_{1, N}=$ $(k / N)\left(1+\mathscr{O}\left(1 / N^{2}\right)\right)$, as it should be on general grounds, and that all $k$-string tensions have a finite limit at zero temperature.

\section{AREA LAW FOR LARGE WILSON LOOPS}

The magnetic field of dyons beyond their cores is Abelian and is a superposition of the Abelian fields of individual dyons. For large Wilson loops we are interested in, it is this superposition field of a large number of dyons that contributes most as they have a slowly decreasing $1 /\left|\mathbf{x}-\mathbf{x}_{i}\right|$ asymptotics, hence the use of the field outside the cores is justified. Owing to self-duality,

$$
\left[B_{i}(\mathbf{x})\right]_{m n}=\left[\partial_{i} A_{4}(\mathbf{x})\right]_{m n}=-\frac{T}{2} \delta_{m n} \partial_{i} \mathrm{v}_{m}(\mathbf{x}),
$$

cf. eq. (16). Since $A_{i}$ is Abelian beyond the cores, one can use the Stokes theorem for the spatial Wilson loop:

$$
\begin{aligned}
W & \equiv \operatorname{Tr} \mathscr{P} \exp i \oint A_{i} d x^{i}=\operatorname{Tr} \exp i \int B_{i} d^{2} \sigma^{i} \\
& =\sum_{m} \exp \left(-i \frac{T}{2} \int d^{2} \sigma^{i} \partial_{i} \mathrm{v}_{m}\right)
\end{aligned}
$$

Eq. (33) may look contradictory as we first use $B_{i}=$ curl $A_{i}$ and then $B_{i}=\partial_{i} A_{4}$. Actually there is no contradiction as the last equation is true up to Dirac string singularities which carry away the magnetic flux. If the Dirac string pierces the surface spanning the loop it gives a quantized contribution $\exp (2 \pi i$. integer $)=1$; one can also use the gauge freedom to direct Dirac strings parallel to the loop surface in which case there is no contribution from the Dirac strings at all.

Let us take a flat Wilson loop lying in the $(x y)$ plane at $z=0$. Then eq. (33) is continued as

$$
\begin{aligned}
W & =\sum_{m} \exp \left(-i \frac{T}{2} \int_{x, y \in \text { Area }} d^{3} x \partial_{z} \mathrm{v}_{m} \delta(z)\right) \\
& =\sum_{m} \exp \left(i \frac{T}{2} \int_{x, y \in \text { Area }} d^{3} x \mathrm{v}_{m} \partial_{z} \delta(z)\right)
\end{aligned}
$$

It means that the average of the Wilson loop in the dyon ensemble is given by the partition function (15) with the source

$$
\sum_{m} \exp \left(i \frac{T}{2} \int d^{3} x \mathrm{v}_{m} \frac{d \delta(z)}{d z} \theta(x, y \in \text { Area })\right)
$$

where $\theta(x, y \in$ Area $)$ is a step function equal to unity if $x, y$ belong to the area inside the loop and zero otherwise.

As in the case of the Polyakov lines the presence of the Wilson loop shifts the argument of the $\delta$-function arising from the integration over the $\mathrm{v}_{m}$ variables, and the ghost determinant cancels exactly the Jacobian from the fluctuations of $\mathrm{w}_{m}$ 's, therefore the classical-field calculation is exact.

One has to solve the non-linear Toda equations on $\mathrm{w}_{m}$ 's with a source along the surface of the loop,

$$
\begin{aligned}
& -\partial^{2} \mathrm{w}_{m}+M^{2}\left(e^{\mathrm{w}_{m}-\mathrm{w}_{m+1}}-e^{w_{m-1}-\mathrm{w}_{m}}\right) \\
& =-2 \pi i \delta_{m m_{1}} \frac{d \delta(z)}{d z} \theta(x, y \in \text { Area }),
\end{aligned}
$$

for all $m_{1}$, plug it into the action $(4 \pi f / N) \mathscr{F}(w)$, and sum over $m_{1}$. In order to evaluate the average of the Wilson loop in a general antisymmetric rank- $k$ representation, one has to take the source in eq. 35 as $-2 \pi i \delta^{\prime}(z)\left(\delta_{m m_{1}}+\ldots+\delta_{m m_{k}}\right)$ and sum over $m_{1}<\ldots<$ $m_{k}$ from 1 to $N$, see eq. (24).

Contrary to the case of the Polyakov lines, one cannot, generally speaking, linearize eq. (35) in $\mathrm{w}_{m}$ but has to solve the non-linear equations as they are. The Toda equations (35) with a $\delta^{\prime}(z)$ source in the r.h.s. define "pinned soliton" solutions $\mathrm{w}_{m}(z)$ that are $1 d$ functions in the direction transverse to the surface spanning the Wilson loop but do not depend on the coordinates $x, y$ provided they are taken inside the loop. Beyond that surface $\mathrm{w}_{m}=0$. Along the perimeter of the loop, $\mathrm{w}_{m}$ interpolate between the soliton and zero. For large areas, 
the action (25) is therefore proportional to the area of the surface spanning the loop, which gives the famous area law for the average Wilson loop. The coefficient in the area law, the 'magnetic' string tension, is found from integrating the action density of the soliton $\mathrm{w}_{m}(z)$ in the $z$ direction.

The exact solutions of Eq. (35) for any $N$ and any representation $k$ have been found in Ref. [2], and the resulting 'magnetic' string tension turns out to be

$$
\sigma_{k, N}=\frac{\Lambda^{2}}{\lambda} \frac{N}{\pi} \sin \frac{\pi k}{N}
$$

which coincides with the 'electric' string tension (31) found from the correlators of the Polyakov lines, for all $k$-strings!

Several comments are in order here.

- The 'electric' and 'magnetic' string tensions should coincide only in the limit $T \rightarrow 0$ where the Euclidean $O(4)$ symmetry is restored. Both calculations have been in fact performed in that limit as we have ignored the temperature-dependent perturbative potential (7). If it is included, the 'electric' and 'magnetic' string tensions split.

- despite that the theory (15) is 3-dimensional, with the temperature entering just as a parameter in the Lagrangian, it "knows" about the restoration of Euclidean $O(4)$ symmetry at $T \rightarrow 0$.

- the 'electric' and 'magnetic' string tensions are technically obtained in very different ways: the first is related to the mass of the elementary excitation of the dual fields $\mathrm{w}_{m}$, whereas the latter is related to the mass of the dual field soliton.

\section{CANCELATION OF GLUONS IN THE CONFINEMENT PHASE}

To prove confinement, it is insufficient to demonstrate the area law for large Wilson loops and the zero average for the Polyakov line: it must be shown that there are no massless gluons left in the spectrum. We give an argument that this indeed happens in the dyon vacuum.

A manifestation of massless gluons in perturbation theory is the Stefan-Boltzmann law for the free energy:

$$
-\frac{T}{V} \log \mathscr{Z}_{\mathrm{SB}}=\frac{F_{\mathrm{SB}}}{V}=-\frac{\pi^{2}}{45} T^{4}\left(N^{2}-1\right) .
$$

It is proportional to the number of gluons $N^{2}-1$ and has the $T^{4}$ behavior characteristic of massless particles. In the confinement phase, neither is permissible: If only glueballs are left in the spectrum the free energy must be $\mathscr{O}\left(N^{0}\right)$ and the temperature dependence must be very weak until $T \approx T_{c}$ where it abruptly rises owing to the excitation of many glueballs.

The nonperturbative free energy corresponding to the minimum of the dyon-induced potential energy as function of the holonomy (21) is

$$
\frac{F_{\text {dyon }}}{V}=-\frac{N^{2}}{2 \pi^{2}} \frac{\Lambda^{4}}{\lambda^{2}} \text {. }
$$

It is $\mathscr{O}\left(N^{2}\right)$ but temperature-independent. We have doubled the minimum from eq. (21) keeping in mind that there are also anti-dyons and assuming that their interactions with dyons is not as strong as the interactions between dyons and anti-dyons separately, as induced by the determinant measure (14), therefore treating dyons and anti-dyons as two independent "liquids". (By the same logic, the string tension (31) has to be multiplied by $\sqrt{2}$ as due to anti-dyons.)

Dyons force the system to have the "most nontrivial" holonomy (5). For that holonomy, the perturbative potential energy (7) is at its maximum equal to

$$
\frac{F_{\text {pert, max }}}{V}=\frac{\pi^{2}}{45} T^{4}\left(N^{2}-\frac{1}{N^{2}}\right) .
$$

The full free energy is the sum of the three terms above.

We see that the leading $\mathscr{O}\left(N^{2}\right)$ term in the StefanBoltzmann law is canceled by the potential energy precisely at the confining holonomy point and nowhere else! In fact it seems to be the only way how $\mathscr{O}\left(N^{2}\right)$ massless gluons can be canceled out of the free energy, and the main question shifts to why does the system prefer the "most nontrivial" holonomy. Dyons seem to answer that question.

\section{DECONFINEMENT PHASE TRANSITION}

As the temperature rises, the perturbative free energy grows as $T^{4}$ and eventually it overcomes the negative nonperturbative free energy (38). At this point, the trivial holonomy for which both the perturbative and nonperturbative free energy are zero, becomes favorable. Therefore an estimate of the critical deconfinement temperature comes from equating the sum of Eq. (38) and Eq. (39) to zero, which gives

$$
T_{c}^{4}=\frac{45}{2 \pi^{4}} \frac{N^{4}}{N^{4}-1} \frac{\Lambda^{4}}{\lambda^{2}} .
$$

As expected, it is stable in $N$. A more robust quantity, both from the theoretical and lattice viewpoints, is the ratio $T_{c} / \sqrt{\sigma}$ where $\sigma$ is the string tension in the fundamental representation, since in this ratio the poorly known 


\begin{tabular}{|c|c|c|c|c|c|}
\hline & $S U(3)$ & $S U(4)$ & $S U(6)$ & $S U(8)$ & $N \rightarrow \infty$ \\
\hline \multirow{2}{*}{$T_{c} / \sqrt{\sigma}$, theory } & 0.6430 & 0.6150 & 0.5967 & 0.5906 & $0.5830+\frac{0.4795}{N^{2}}+\frac{0.5006}{N^{4}}+\ldots$ \\
\hline \multirow{2}{*}{$T_{c} / \sqrt{\sigma}$, lattice } & $0.6462(30)$ & $0.6344(81)$ & $0.6101(51)$ & $0.5928(107)$ & $0.5970(38)+\frac{0.449(29)}{N^{2}}($ fit $)$ \\
\hline
\end{tabular}

parameters $\Lambda$ and $\lambda$ cancel out:

$$
\frac{T_{c}}{\sqrt{\sigma}}=\left(\frac{45}{4 \pi^{4}} \frac{\pi^{2} N^{2}}{\left(N^{4}-1\right) \sin ^{2} \frac{\pi}{N}}\right)^{\frac{1}{4}} \stackrel{N \rightarrow \infty}{\longrightarrow} \frac{1}{\pi}\left(\frac{45}{4}\right)^{\frac{1}{4}} .
$$

In the Table, we compare the values from Eq. (41) to those measured in lattice simulations of the pure $S U(N)$ gauge theories [28]; there is a surprisingly good agreement. A detailed study of the thermodynamics of the phase transition will be published elsewhere.

\section{AN EXCEPTIONAL GIFT}

It is illuminating to go beyond the $S U(N)$ gauge groups and consider the YM theory based on $e$.g. the exceptional group $G(2)$. It has rank 2 and is similar to the $S U(3)$ group but has only a trivial center, meaning that only a unity element commutes with all other group elements (in $S U(N)$ there are $N$ such matrices being $N$-roots of unity; they form the group center $Z_{N}$ ). Since confinement in $S U(N)$ is often associated with the symmetric distribution of the Polyakov line eigenvalues with respect to the $Z_{N}$ permutations, and the deconfinement transition is associated with the spontaneous breaking of $Z_{N}$ symmetry, while neither is a feature of $G(2)$, questions have been raised whether there is at all confinement in $G(2)$, leave alone the confinement-deconfinement phase transition.

However, recent numerical simulations of $G(2)$ performed by three groups [29] showed that there is confinement at low $T$ and a first-order deconfinement transition. By confinement we mean here the zero average of the Polyakov line in the lowest 7-dimensional representation. These findings pose difficulties for the confinement scenarios based on the center of the group, e.g. on center vortices.

The dyon scenario works, however, exceptionally well it this case, too. We have repeated all the steps described in this paper but applied to the $G(2)$ group. There are three types of fundamental dyons, like in $S U$ (3), but neutral clusters are formed by four; overall neutrality requires that one type of dyons have to enter twice more often than the two others. The minimum of the free energy for the dyon ensemble lies exactly at the holonomy cor- responding to the zero Polyakov line. At a critical temperature there is a first-order transition to a phase with a nonzero average Polyakov line [30].

We stress that these results are sensitive to the dynamics as they are not in the least enforced by symmetry. They provide strong support to the dyon scenario of confinement. It should be added that instantons with nontrivial holonomy and dyons have been directly observed in lattice simulations [31].

\section{SUMMARY}

What happens in the semiclassical approximation based on dyons, can be summarized as follows:

- The ensemble of dyons favors dynamically the confining value of the holonomy. This is almost clear, given that the weight is proportional to the product of individual actions of fundamental dyons, and it is maximal when the actions are equal. Such holonomy corresponds to the zero of the Polyakov line

- Dyons form a sort of Coulomb plasma (but an exactly solvable variant of it) with an appearance of the Debye mass both for "electric" and "magnetic" (dual) photons. The first gives rise to the exponential fall-off of the correlation of two Polyakov lines, i.e. to the linear heavy-quark potential, the second yields the area law for spatial Wilson loops

- $\mathscr{O}\left(N^{2}\right)$ massless gluons cancel out from the free energy, and only massive (string?) excitations are left.

The reason why a semiclassical approximation works well for strong interactions (where all dimensionless quantities are, generally speaking, of the order of unity) is not altogether clear. A possible justification has been outlined in the Introduction: After UV renormalization is performed about the classical saddle points and the scale parameter $\Lambda$ appears as the result of the dimensional transmutation, further quantum corrections to the saddle point are a series in the running 't Hooft coupling $\lambda$ whose argument is typically the largest scale in the theory, in this case $\max \left(T, n^{1 / 4}\right)$ where $n$ is the $4 d$ density of dyons. An estimate shows that the running $\lambda$ is 
between $1 / 4$ at zero temperature and $1 / 7$ or less at critical temperature. Therefore, although these numbers are "of the order of unity", in practical terms they indicate that high order loop corrections are not too large. Let us recall that quite an accurate computation of anomalous dimensions in critical phenomena from the $\varepsilon$-expansion by Fisher and Wilson [32] is based on truncating the Taylor expansion in $\varepsilon$ at the first couple of terms, where $\varepsilon=1$ or sometimes $2 \square$

Unfortunately, approximations made in Ref. [2] and reproduced above are not limited to neglecting higher loop corrections. We have (i) ignored dyon interactions induced by the small oscillation determinant over nonzero modes (although we did take into account that it renormalizes the gauge coupling giving rise to the scale parameter $\Lambda$, and that it leads to the perturbative potential energy as function of the holonomy), (ii) neglected the interactions of dyons of opposite duality, treating them as two noninteracting "liquids", (iii) conjectured a simple form of the dyon measure which may be incorrect when two same-kind dyons come close. Although certain justification for these approximations can be put forward [2] it is desirable not to use them at all, and that may be possible.

\section{ACKNOWLEDGMENTS}

This work has been supported in part by Russian Government grants RFBR-06-02-16786 and RSGSS5788.2006.2.

\section{REFERENCES}

1. H. Fritzsch, M. Gell-Mann and P. Minkowski, Phys.Lett. B47, 365 (1973)

2. D. Diakonov and V. Petrov, Phys. Rev. D76, 056001 (2007), arXiv:0704.3181

3. D.J. Gross, R.D. Pisarski and L.G. Yaffe, Rev. Mod. Phys. 53, 43 (1981)

4. N. Weiss, Phys. Rev. D24, 475 (1981); Phys. Rev. D25, 2667 (1982)

5. D. Diakonov and M. Oswald, Phys. Rev. D70, 105016 (2004), arXiv:hep-ph/0403108

6. N.M. Davies, T.J. Hollowood, V.V. Khoze and M.P. Mattis, Nucl. Phys. B559, 123 (1999), arXiv:hep-th/9905015

7. D. Diakonov, Prog. Part. Nucl. Phys. 51, 173 (2003), arXiv:hep-ph/0212026

8. E.B. Bogomolny, Yad. Fiz. 24, 861 (1976) [Sov. J. Nucl. Phys. 24, 449 (1976)]; M.K. Prasad and C.M. Sommerfield, Phys. Rev. Lett. 35, 760 (1975)

\footnotetext{
${ }^{1}$ One of us (D.D.) takes the opportunity to thank Michael Fisher and Valery Pokrovsky for a discussion of this numerical miracle.
}

9. D. Diakonov and V. Petrov, Phys. Rev. D67, 105007 (2003), arXiv:hep-th/0212018

10. T.C. Kraan and P. van Baal, Phys. Lett. B428, 268 (1998), arXiv:hep-th/9802049. Nucl. Phys. B533, 627 (1998), arXiv:hep-th/9805168

11. K. Lee and C. Lu, Phys. Rev. D58, 025011 (1998), arXiv:hep-th/9802108

12. K. Lee and P. Yi, Phys. Rev. D56, 3711 (1997), arXiv:hep-th/9702107

13. T.C. Kraan and P. van Baal, Phys. Lett. B435, 389 (1998), arXiv:hep-th/9806034

14. F. Bruckmann, D. Nogradi and P. van Baal, Acta Phys. Polon. B34, 5717 (2003), arXiv:hep-th/0309008

15. B.J. Harrington and H.K. Shepard, Phys. Rev. D17, 2122 (1978); Phys. Rev. D18, 2990 (1978)

16. A. Belavin, A. Polyakov, A. Shvarts and Yu. Tyupkin, Phys. Lett. 59, 85 (1975)

17. D. Diakonov, N. Gromov, V. Petrov and S. Slizovskiy, Phys. Rev. D70, 036003 (2004), arXiv:hep-th/0404042 N. Gromov, in: Proc. NATO Advanced Study Institute and EU Hadron Physics 13 Summer Institute, St. Andrews, Scotland, 22-29 Aug 2004, p. 411, arXiv:hep-th/0701192

18. S. Slizovkiy, Phys. Rev. D76, 085019 (2007), arXiv:0707.0851 [hep-th]

19. T.C. Kraan, Commun. Math. Phys. 212, 503 (2000), arXiv:hep-th/9811179

20. D. Diakonov and N. Gromov, Phys. Rev. D72, 025003 (2005), arXiv:hep-th/0502132

21. M.F. Atiyah and N.J. Hitchin, The Geometry and Dynamics of Magnetic Monopoles, Princeton University Press (1988)

22. F.A. Berezin, Second Quantization Method, Nauka, Moscow (1965)

23. A. Polyakov, Nucl. Phys. B120, 429 (1977)

24. M. Shifman, Acta Phys. Polon. B6, 3805 (2005), arXiv:hep-th/0510098

25. M.R. Douglas and S.H. Shenker, Nucl. Phys. B447, 271 (1995); A. Hanany, M. Strassler and A. Zaffaroni, Nucl. Phys. B513, 87 (1998), arXiv:hep-th/9707244

26. L. Del Debbio, H. Panagopoulos, P. Rossi and E. Vicari, JHEP 0201, 009 (2002), arXiv:hep-th/0111090

27. B. Lucini, M. Teper and U. Wenger, JHEP 0406, 012 (2004), arXiv:hep-lat/0404008

28. B. Lucini, M. Teper and U. Wenger, JHEP 0401, 061 (2003), arXiv:hep-lat/0307017, B. Lucini, M. Teper and U. Wenger, arXiv:hep-lat/0502003

29. J. Greensite, , K. Langfeld, S. Olejnik, H. Reinhardt and T. Tok, Phys. Rev. D75, 034501 (2007) arXiv:hep-lat/0609050; M. Pepe and U.-J. Wiese, Nucl. Phys. B76, 21 (2007), arXiv:hep-lat/0610076, G. Cossu, M. D'Elia, A. Di Giacomo, B. Lucini and C. Pica, JHEP 0710, 100 (2007), arXiv:0709.0669 [hep-lat]

30. D. Diakonov and V. Petrov, in preparation

31. V.G. Bornyakov, E.-M. Ilgenfritz, B.V. Martemyanov, S.M. Morozov, M. Müller-Preussker and A.I. Veselov, Phys. Rev. D76, 054505 (2007), arXiv:0706.4206 [hep-lat]

32. K. Wilson and M. Fisher, Phys. Rev. Lett. 28, 240 (1972). 